\title{
Planar Laser-Induced Iodine Fluorescence Measurements in Rarefied Hypersonic Flow
}

\author{
Eric Cecil* and James C. McDaniel* \\ *Department of Mechanical and Aerospace Engineering, University of Virginia, Charlottesville, VA 22903, USA
}

\begin{abstract}
A planar laser-induced fiuorescence (PLIF) technique is discussed and applied to measurement of time-averaged values of velocity and temperature in an $\mathrm{I}_{2}$-seeded $\mathrm{N}_{2}$ hypersonic free jet facility. Using this technique, a low temperature, non-reacting, hypersonic flow over a simplified model of a reaction control system (RCS) was investigated. Data are presented of rarefied Mach 12 flow over a sharp leading edge flat plate at zero incidence, both with and without an interacting jet issuing from a nozzle built into the plate. The velocity profile in the boundary layer on the plate was resolved. The slip velocity along the plate, extrapolated from the velocity profile data, varied from nearly $100 \%$ down to $10 \%$ of the freestream value. These measurements are compared with results of a DSMC solution. The velocity variation along the centerline of a jet issuing from the plate was measured and found to match closely with the correlation of Ashkenas and Sherman. The velocity variation in the oblique shock terminating the jet was resolved sufficiently to measure the shock wave thickness.
\end{abstract}

\section{IODINE FLUORESCENCE MEASUREMENT TECHNIQUE}

Numerous methods have been reported in the literature for measurement of thermodynamic properties in supersonic flows using laser-induced fluorescence. This paper presents results of a technique based on narrowband excitation of fluorescence from nuclear hyperfine components in the $B \leftrightarrow X$ electronic transition of molecular iodine. This approach was developed to study low temperature flows of $I_{2}$-seeded $N_{2}$ gas. A tunable single frequency laser beam is expanded and collimated into a thin sheet and passed into a low density wind tunnel flow, causing $\mathrm{I}_{2}$ molecules to fluoresce within a selected planar section of the flow-field. A series of images are taken of the fluorescence with a charge-coupled device (CCD) array as the laser frequency is incrementally tuned over selected lines in the $I_{2}$ absorption spectrum. The fluorescence signal versus frequency is thereby obtained for any point in the plane of the laser sheet, within the resolution of the digital image. These absorption spectra can provide information on the local thermodynamic conditions in the flow. This paper describes PLIF measurements of velocity and temperature made in a low density, low temperature, hypersonic flow over a simplified model of an RCS [1], intended to support assessment of a hybrid numerical solution method for such flows described in $[2,3]$.

The fluorescence signal produced by $\mathrm{I}_{2}$ vapor (in a stationary, low pressure carrier gas) from a single transition line $i$, centered at frequency $v_{0, i}$, due to excitation at frequency $v$, is given by $[1,4]$

$$
S_{f_{i}}(v)=C I n_{\mathrm{I}_{2}} f_{v^{\prime \prime} J^{\prime \prime}}(T) q_{v^{\prime} v^{\prime \prime}} g_{\mathrm{hf}} S_{\mathrm{hf}, i} \frac{S_{J^{\prime} J^{\prime \prime}}}{2 J^{\prime \prime}+1} \frac{V\left(v, v_{0, i}, P, T\right)}{\Delta v_{D}(T)},
$$

where $C$ is a constant representing signal collection efficiency and molecular constants, $I$ is excitation source intensity, $n_{\mathrm{I}_{2}}$ is $\mathrm{I}_{2}$ number density, $P$ is carrier gas pressure, $T$ is temperature, $f_{v^{\prime \prime}} j^{\prime \prime}$ is the Boltzmann population of the ground vibrational-rotational state $v^{\prime \prime} J^{\prime \prime}, q_{v^{\prime} v^{\prime \prime}}$ is the Franck-Condon factor, $g_{\mathrm{hf}}$ is the hyperfine degeneracy, $S_{\mathrm{hf}, i}$ is the hyperfine line strength, $S_{J^{\prime} J^{\prime \prime}}$ is the Hönl-London rotational line strength, and $\Delta v_{D}(T)$ is the Doppler line width. The Voigt function $V\left(v, v_{0, i}, P, T\right)$ is a convolution integral of Gaussian and Lorentzian lineshape contributions. Collisional quenching of the excited state has been assumed to be negligible, so that the fluorescence efficiency is unity.

The measured fluorescence signal is the resultant of fluorescence from all excited absorption lines, each of which contributes an amount given by (1). Because broadening mechanisms cause overlapping contributions from closely spaced transitions, the net signal due to excitation at frequency $v$ from $N_{t}$ excited transitions is $S_{f}(v)=\Sigma_{i}^{N_{t}} S_{f_{i}}(v)$. 


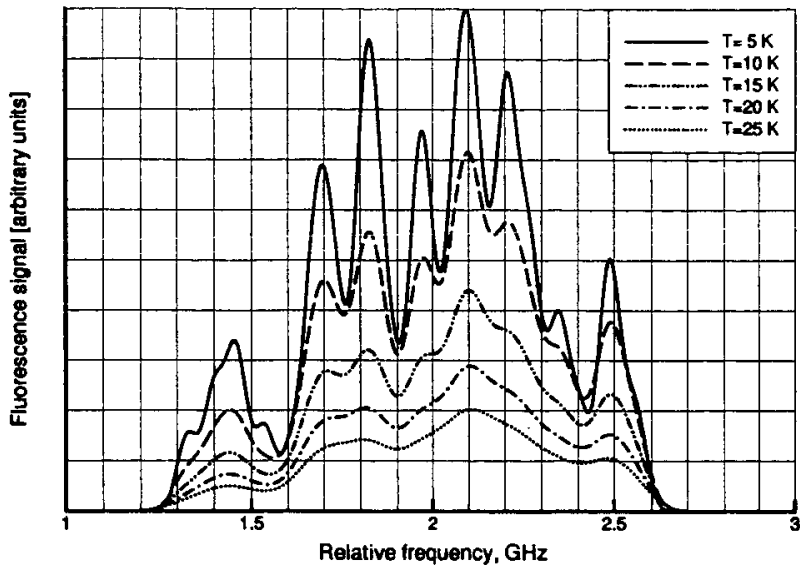

FIGURE 1. Computed P13,R15 hyperfine lineshape at low temperatures, plotted at very low constant pressure.

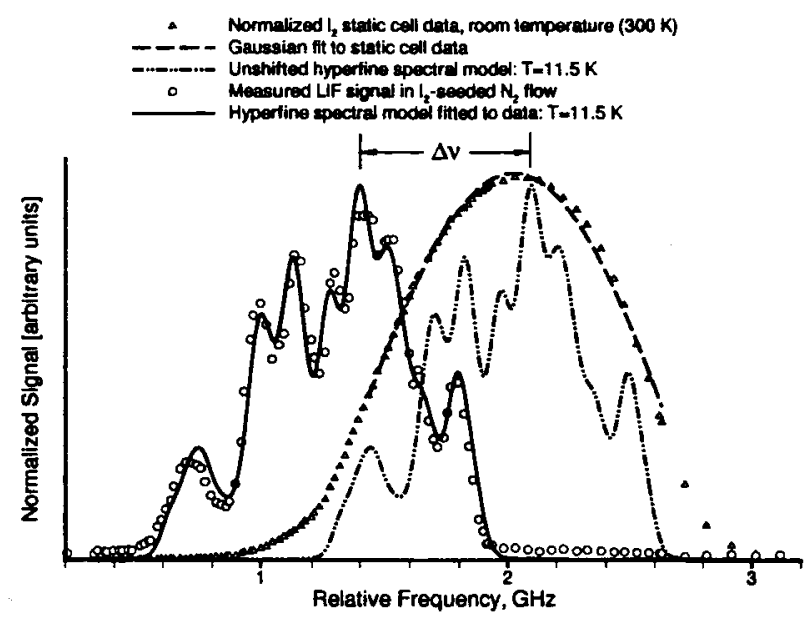

FIGURE 2. Determination of temperature from lineshape and velocity component from frequency shift $\Delta v$.

Due to nuclear electric quadrupole coupling and magnetic spin-rotation interactions in the $\mathrm{I}_{2}$ molecule, the P13 and R15 rotational lines of the (43-0) vibrational band each have hyperfine structure consisting of 21 component lines $[5,6]$. The frequencies of these 42 hyperfine components span $1.23 \mathrm{GHz}$ and are interleaved, giving the appearance of a single blended line in the presence of significant broadening, as in $\mathrm{I}_{2}$ vapor at room temperature. Lorentz (pressure) broadening due to $\mathrm{I}_{2}-\mathrm{N}_{2}$ collisions is largely absent at the low pressures encountered in this study. The observed broadening arises almost entirely due to thermal (Doppler) broadening. Below $50 \mathrm{~K}$, thermal broadening is sufficiently low to hint at the underlying hyperfine structure. The computed (low pressure) P13,R15 lineshape $S_{f}(v)$ is plotted at several temperatures from $5 \mathrm{~K}$ to $25 \mathrm{~K}$ in fig. 1. Because of the irregular, close spacing of the hyperfine components, only four distinct peaks are discernable at $25 \mathrm{~K}$. At 10 to $15 \mathrm{~K}$, seven distinct peaks can be distinguished. Further peaks appear in the lineshape at $5 \mathrm{~K}$. In a free jet expansion from ambient temperature, this combination of low pressure and temperature is typical. The temperature sensitivity of the transition lineshape makes possible temperature determination by nonlinear least-squares regression fitting of the hyperfine spectral model to measured spectral data.

When the excited gas has a mean speed in the direction of laser propagation, $u$, the observed lineshape will be Doppler-shifted by an amount $u / \lambda$, where $\lambda$ is the excitation wavelength. (At low pressure, the "impact shift" from collisional perturbation of molecular energy levels may be neglected.) The velocity component $u$ is found by measurement of the frequency shift, $\Delta v$, of the lineshape relative to a reference spectrum measured simultaneously in a stationary cell of $\mathrm{I}_{2}$ vapor at room temperature. The determination of $\Delta v$ from typical data is illustrated in fig. 2 . In practice, the $\Delta v$ is determined as part of the nonlinear regression fitting process, along with the temperature. Planar velocity measurements require two spectral data sets in which the laser sheet enters at different angles to establish two independent velocity components.

A Spectra-Physics BeamLok 2080A-15 argon ion laser operating at $514 \mathrm{~nm}$ was used to excite $\mathrm{I}_{2}$ fluorescence in the present experiments. A temperature-stabilized, air-spaced intracavity etalon eliminated all but a single longitudinal mode of the laser. The etalon tilt angle, which can shift the etalon loss curve to select the longitudinal mode, was controlled via a geared stepper motor attachment. Etalon tuning between longitudinal modes of the laser ( $84 \mathrm{MHz}$ increments) is insufficient to resolve fine detail in the hyperfine $I_{2}$ spectrum, which has thermally broadened line widths on the order of $80 \mathrm{MHz}$ at $10 \mathrm{~K}$. Fine frequency scanning of the laser (typically in $20 \mathrm{MHz}$ steps) was accomplished by varying the laser cavity length. The "BeamLok" system of the laser incorporates a piezoelectric translator which can move the laser output mirror up to $1.5 \mu \mathrm{m}$, thereby changing the cavity length and allowing continuous scanning over a range of a few hundred $\mathrm{MHz}$ as the applied voltage is varied. Because that range is limited, fine scanning is done in conjunction with intermittent etalon tilt adjustments to scan the full range of the laser gain profile.

For frequency measurement, the laser beam is sampled by a Fabry-Perot scanning interferometer (Spectra-Physics model 450) and a static $I_{2}$ cell. Relative frequency increments between camera exposures are measured with the interferometer, which has a bandwidth of $10 \mathrm{MHz}$. Fluorescence in the static cell is measured with a photodetector to provide an absolute frequency reference.

Images were taken with a Photometrics $\mathrm{CH} 210$ liquid nitrogen-cooled camera system using a PM512 CCD having 


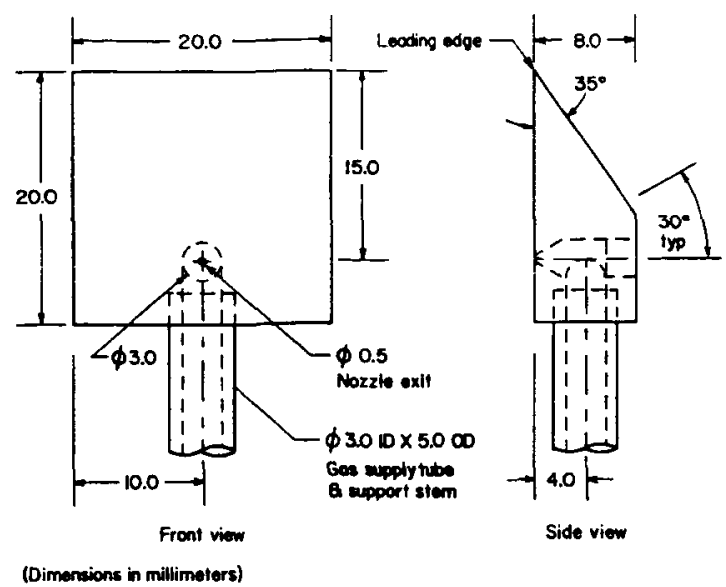

FIGURE 3. A dimensional drawing of the quartz RCS model.

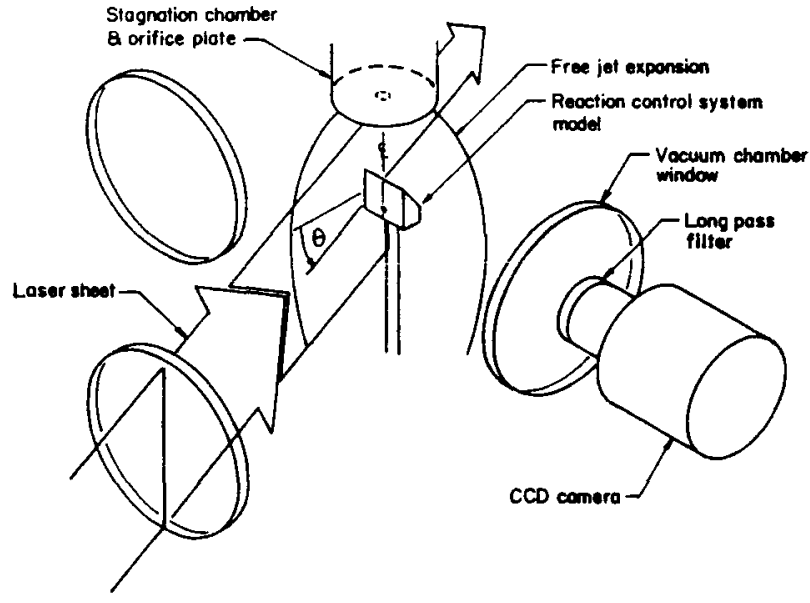

FIGURE 4. Experimental set-up for LIF imaging of the simulated RCS flowfield in the low density wind tunnel.

a $516 \times 516$ array of $20 \mu \mathrm{m}$ square pixels (yielding a $1.03 \mathrm{~cm} \times 1.03 \mathrm{~cm}$ image plane). This system allows acquisition of low noise, 14-bit, highly linear, quantitative images under low light conditions. An $85 \mathrm{~mm} f / 1.4$ Nikon lens with an $8 \mathrm{~mm}$ extension ring (to reduce the minimum focusing distance) was used with a $570 \mathrm{~nm}$ long pass Schott glass filter which blocked scattered laser light. The camera was placed with a subject-to-image plane distance of about $64 \mathrm{~cm}$, resulting in a spatial resolution of $7.2( \pm .05)$ pixels $/ \mathrm{mm}$. Due to exposure times of $20-75 \mathrm{sec}$. for each image, the measurements yield time-averaged quantities.

An underexpanded free jet was produced by the expansion of $\mathrm{I}_{2}$-seeded $\mathrm{N}_{2}$ gas across a thin circular orifice of diameter $D=2 \mathrm{~mm}$ into a continuously evacuated vacuum chamber. Stagnation properties of the flow are measured in a settling chamber immediately upstream of the orifice. Gas issuing from the sonic condition at the orifice overexpands into a source-like hypersonic flow which forms a barrel-shaped shock wave and terminates at a nearly normal shock known as the Mach disk. The free jet core serves as the hypersonic wind tunnel test section.

The interaction of a hypersonic freestream with an RCS is simulated by introducing a model of the RCS inside the jet core, upstream of the Mach disk location in the undisturbed free jet. The RCS model consists of a flat plate which has a small circular nozzle exit of diameter $d=0.5 \mathrm{~mm}$ on its surface from which a transverse jet may be injected from a $60^{\circ}$ converging conical nozzle. The model was constructed of optical quality fused quartz to minimize scattered laser light. A diagram of the model geometry is shown in fig. 3. The flat surface of the model was centered parallel to the symmetry axis of the freestream jet, so as to be at zero incidence. The leading edge of the model was located approximately $41 \mathrm{~mm}(20.5 \mathrm{D})$ downstream of the orifice, where the effective freestream Mach number at the leading edge, $M_{L E} \approx 11.9$, based on the correlation of Ashkenas and Sherman [7].

For the data presented here, the laser beam was expanded and collimated into a thin, $14 \mathrm{~cm}$ wide sheet and passed through a window in the low density wind tunnel to excite fluorescence in the central symmetry plane of the threedimensional flow over the model. A schematic of the PLIF imaging set-up is shown in fig. 4.

\section{MEASUREMENTS}

A narrowband PLIF image of flow over the flat plate model is shown in figure 5. Light areas in the image indicate spatial fluorescence intensity patterns, corresponding to a laser sheet incidence of $\theta=28.0^{\circ}$ in fig. 4 . Aside from the model outline (which appears because the filter could not completely block scattered light), several flow structures are evident, such as the oblique shock on the plate, the outer barrel shock, and a shock connecting the two which is analogous to the Mach disk in an undisturbed free jet. The pronounced finger-like pattern in the upper right of the image is due to a Doppler shift in the excitation frequency which depends on the laser sheet incidence angle and the local flow velocity vector. As a result, fluorescence corresponding to the hyperfine components of the P13,R15 transition produces intense rays running parallel to streamlines in the "free stream" of the flow. A series of over a hundred such images, with varying excitation frequency, are required to resolve the spectrum at each pixel. A second 


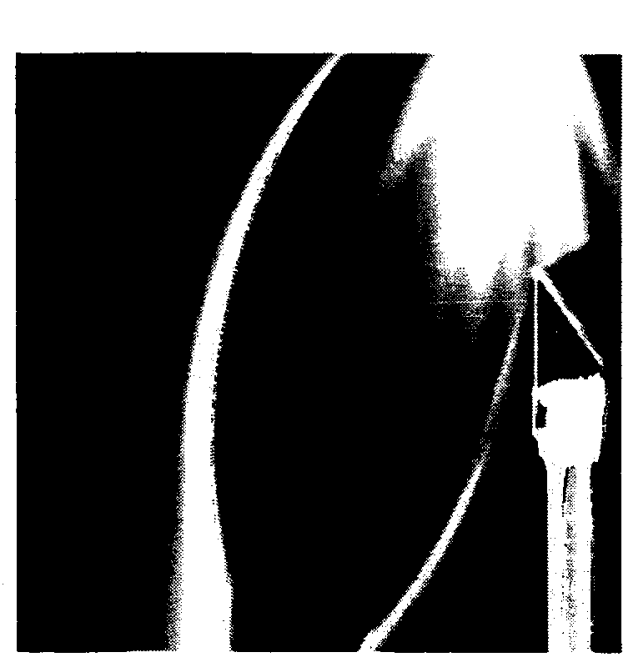

FIGURE 5. $I_{2}$ PLIF image of hypersonic flow over the flat plate model.

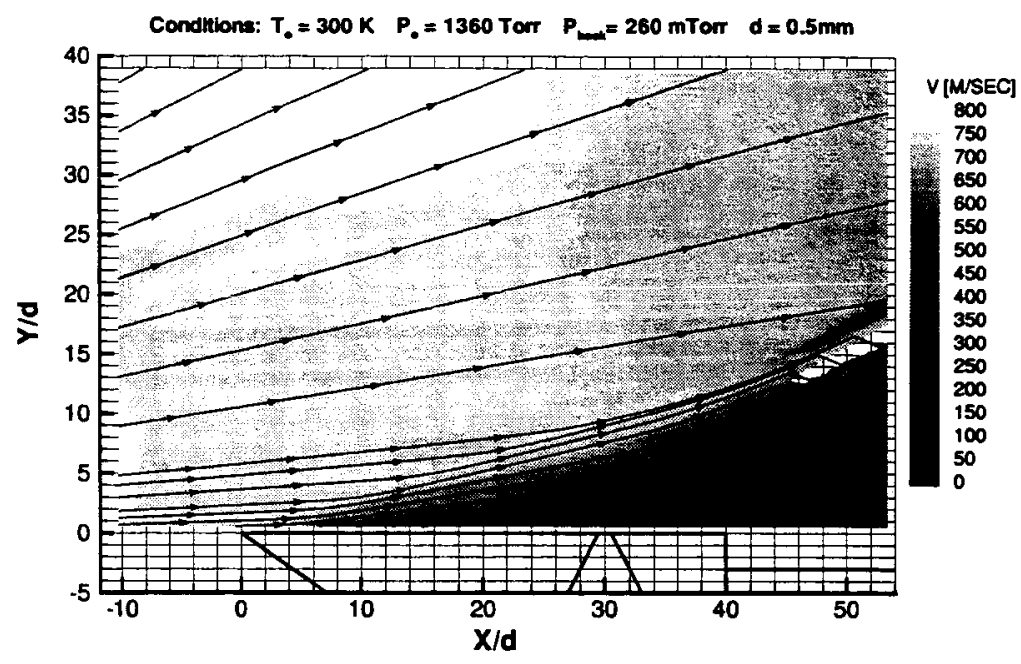

FIGURE 6. Measured velocity contours and streamlines on the centerplane of the hypersonic $\left(M_{L E} \approx 11.9\right.$ ) flow over the flat plate.

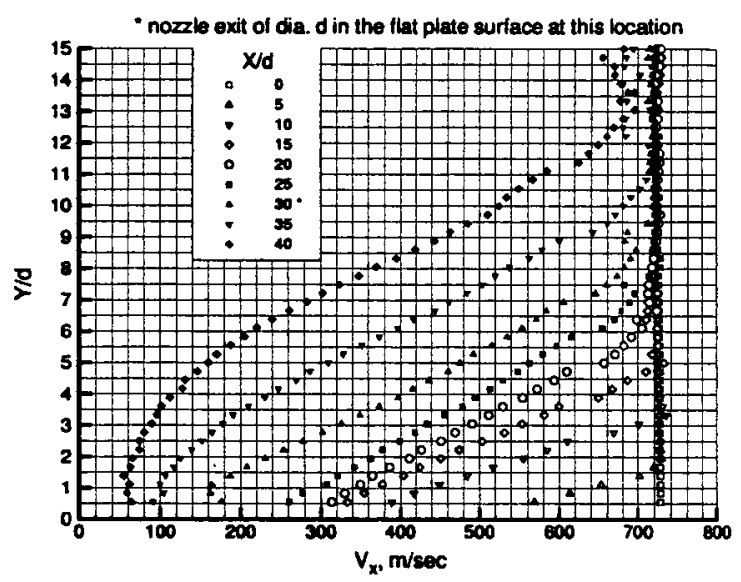

FIGURE 7. Velocity profiles measured at several stations along the flat plate surface.

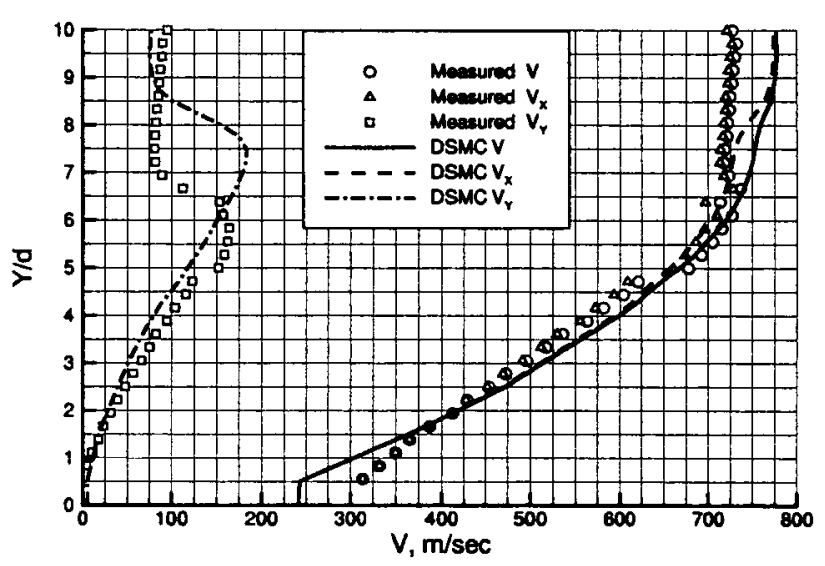

FIGURE 8. Comparison of measured velocity profile at $X / d=20$ with a DSMC computation [8].

set of images was made with $\theta=-31.4^{\circ}$ to fully determine the planar velocity components.

Measured velocities in the flow over the plate are shown in fig. 6 . The $X-Y$ coordinates originate at the leading edge of the plate and are aligned with the surface. Streamlines shown in the figure were produced from Cartesian velocity components determined from the non-orthogonal velocity component measurements. The location of the shock wave was estimated by drawing a straight line through the locus of points where streamlines first change direction. This location indicated a shock wave angle of approximately $18^{\circ}$, which is in agreement with the angle indicated by fluorescence intensity of the oblique shock in fig. 5 . This angle considerably exceeds the inviscid flow Mach wave angle $\mu=\sin ^{-1} 1 / M_{L E}=4.8^{\circ}$. The discepancy is due to the presence of a thick boundary layer on the plate. According to oblique shock theory for an inviscid Mach 12 flow, an effective angular displacement of roughly $13-14^{\circ}$ of the flow by the boundary layer is necessary to produce the $18^{\circ}$ shock angle. It may therefore be expected that viscous effects will dominate the velocity profiles on the plate.

Tangential velocity profiles measured at nine equally spaced stations along the plate, from the leading edge to the trailing edge, are shown in fig. 7. The large velocity gradients present throughout each profile (up to the freestream) suggest a strong interaction of viscous and compressibility effects. Upstream of the nozzle exit at $X / d=30$, these gradients appear linear almost all the way from the surface out to the free stream. There is no clear delineation of the boundary layer from an inviscid layer behind the shock. 

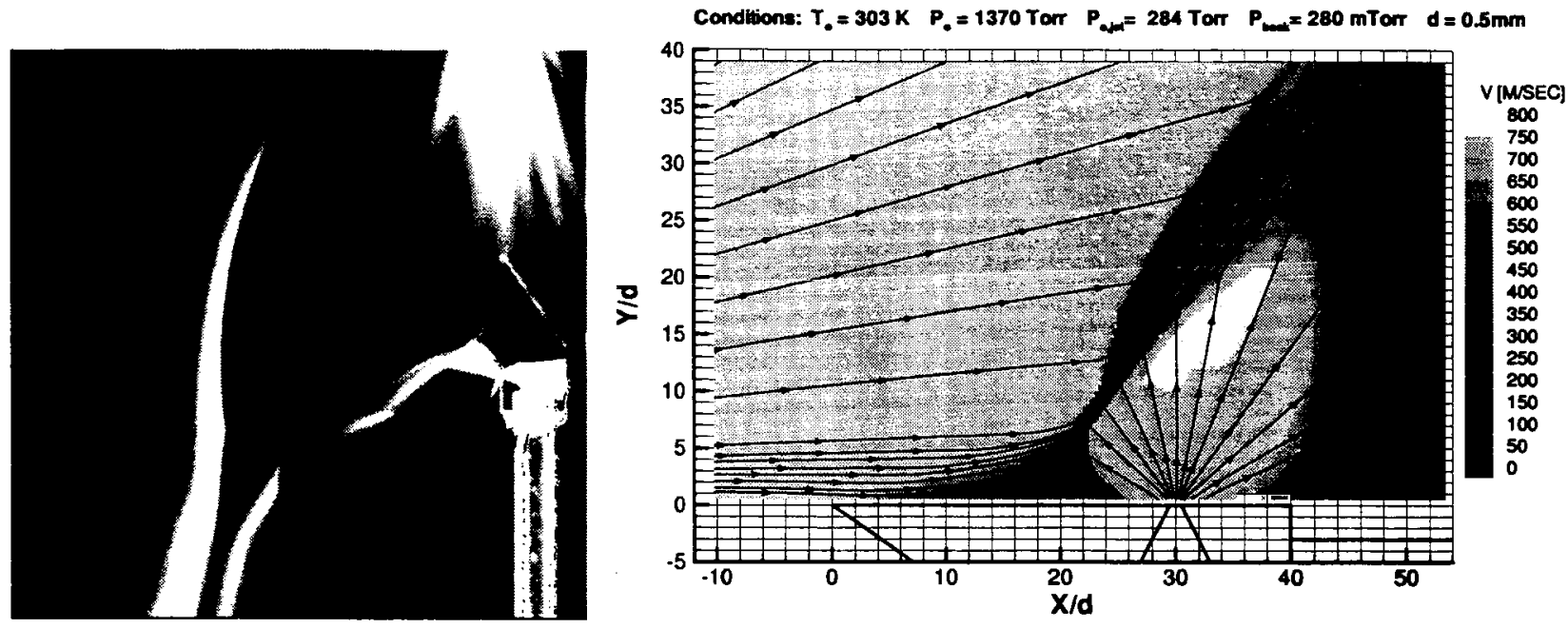

FIGURE 9. $I_{2}$ PLIF image of the RCS jetfreestream interaction.

FIGURE 10. Measured velocity contours and streamlines on the centerplane of the hypersonic $\left(M_{L E} \approx 11.9\right)$ flow over the RCS jet model.

Because the flow is rarefied, "slip flow" (non-zero velocity at the plate surface) can occur. It was not possible to measure velocity closer to the surface than about $d / 2$. However, if the linearity in the velocity profile is extrapolated to the surface, the data indicate a slip velocity of about $270 \mathrm{~m} / \mathrm{s}$ at $X / d=20$, for example. The extrapolated slip velocity decreases with increasing distance $X$ from the leading edge along the plate.

Upstream of the jet exit on the plate $(X / d<30)$, the profiles behind the shock appear nearly linear. The presence of the nozzle exit on the plate surface appears to be the cause of disturbance in the velocity profiles at $X / d>30$, although the nozzle was not in use. Despite the source flow behavior of the wind tunnel free jet, the profiles all agree closely on the freestream tangential velocity $(\approx 725 \mathrm{~m} / \mathrm{s})$.

A preliminary DSMC computation of this flow configuration [8] was made by Chris Glass at the Aerothermodynamics Branch of NASA Langley Research Center, who served as a technical monitor on this research. The computation modeled the flow of the $\mathrm{N}_{2}$ carrier gas molecules only. The velocity profile at the location $X / d=20$ on the flow centerplane has been extracted from the three-dimensional DSMC flow solution and plotted in fig. 8 for comparison with the PLIF measurements. The boundary layer development in the DSMC solution is qualitatively very similar to what is observed in the PLIF data. The disagreement appears to be a direct result of a slightly greater shock angle of $20^{\circ}$ predicted by the DSMC.

A fluorescence image of a simulated RCS flow is shown in fig. 9. The RCS jet interacts with the freestream to produce a bow shock wave. Velocity measurements for this flow are shown in fig. 10. For these measurements, the nominal flow parameters of the preceding flat plate flow were maintained. The flow was altered only by the injection of a transverse jet from the conical nozzle built into the plate, simulating flow from a reaction control jet. In this measurement the ratio of the transverse jet total pressure to the freestream total pressure, $P_{o \text { jet }} / P_{o}=0.208$. The transverse jet was operated at $P_{o \text { jet }} d=142$ torr-mm and a Reynolds number at the sonic condition, $R e_{d}^{*} \cong 2800$.

Near the leading edge, the flow retains the character of the flat plate flow. In this case however, the flow comes to a stagnation region in the boundary layer upstream of the transverse jet. The streamline pattern in this area of the flow shows two regions of opposite vorticity which arise from the jet interaction with the freestream. This qualitatively corroborates the presence of two counter-rotating vortices in a DSMC simulation of a similar flow in ref. [2].

The transverse jet structure is similar to an undisturbed free jet, but terminates asymmetrically at a curved oblique shock surface due to interaction with the oncoming freestream. The variation of velocity along the centerline of the transverse jet is plotted in fig. 11. These measurements are in good agreement with the isentropic expansion velocity of $\mathrm{N}_{2}$ predicted by the Ashkenas and Sherman relation [7] and plotted in the same figure. In undergoing an expansion, a heavy trace gas can exhibit "slip" relative to a light carrier gas, due to the lower terminal velocity characteristic of the heavier molecule. The agreement of the measured $\mathrm{I}_{2}$ trace gas velocity with the predicted centerline velocity of the $\mathrm{N}_{2}$ gas indicates no significant slip of the $\mathrm{I}_{2}$ molecules relative to the $\mathrm{N}_{2}$ in the jet under these conditions.

The velocity variation through the oblique shock wave which follows the expansion is also resolved in fig. 11 . Taking the shock angle as $43^{\circ}$, the measured shock thickness, $\delta=0.42 \mathrm{~mm}$, based on the maximum velocity gradient. 


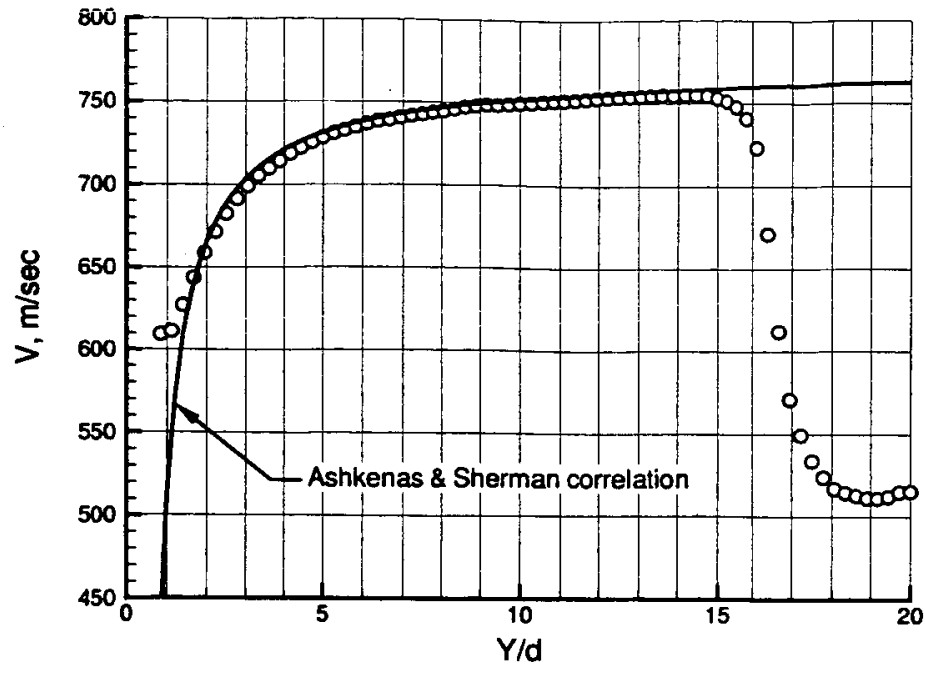

FIGURE 11. Measured velocity along the transverse jet centerline.
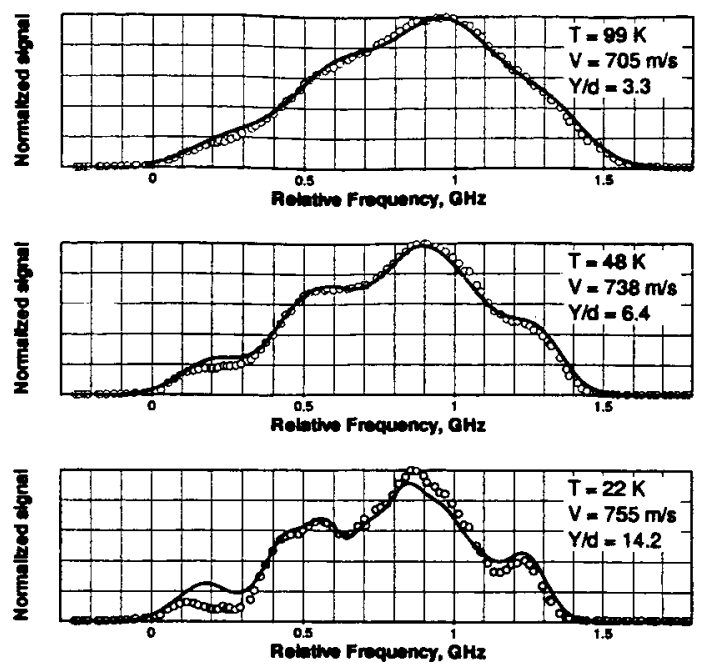

FIGURE 12. Measured $P 13, R 15$ spectra $\left(\theta=30^{\circ}\right)$ at 3 points on the transverse jet centerline.

The mean free path just upstream of the shock, $\lambda_{1} \cong 0.34 \mathrm{~mm}$, based on the inviscid correlation in [7]. The reciprocal thickness $\lambda_{1} / \delta=0.81$ is notably higher than the value of 0.43 reported by Robben and Talbot for a Mach 7 normal shock in $\mathrm{N}_{2}$ [9]. The ratio of the upstream and downstream normal velocities across the shock (not shown in the figure) was roughly $520 / 90=5.77$, reasonably close to the theoretical value of 5.46 for a Mach 7.1 normal shock.

Spectra acquired at three points along the centerline of the transverse jet are shown in fig. 12 along with the fitted spectral model at the indicated temperature. The spatial variation in the measured temperature, $T \propto Y^{-1}$, is significantly higher than predicted by the isentropic correlation of ref. [7]. This is inconsistent with the good agreement of the temperature measurement of $11.5 \mathrm{~K}$ in the freestream $1 \mathrm{~mm}$ upstream of the plate leading edge (this spectrum is shown in fig. 2) compared to the isentropic expansion prediction of $10.3 \mathrm{~K}$.

\section{ACKNOWLEDGEMENTS}

This research was supported by the NASA Graduate Student Researchers Program and grant NAG 1-01051 from the Aerothermodynamics Branch at NASA Langley Research Center.

\section{REFERENCES}

1. D. E. Cecil, Planar Laser-Induced Iodine Fluorescence Measurements in Rarefied Hypersonic Flow Over a Reaction Control Jet Model in a Free Jet Wind Tunnel, M.S. Thesis, University of Virginia, Jan. 2004.

2. C. E. Glass, and G. J. LeBeau, Numerical Study of a Continuum Sonic Jet Interacting With a Rarefied Flow, AIAA paper 97-2536, June 1997.

3. C. E. Glass, Numerical Study of Rarefied Hypersonic Flow Interacting With a Continuum Jet, Ph.D. dissertation, Penn. State, Aug. 1999.

4. J. C. McDaniel, Investigation of Laser-Induced Iodine Fluorescence for the Measurement of Density in Compressible Flows, Ph.D. dissertation, Stanford, Dec. 1981.

5. M. D. Levenson and A. L. Schawlow, "Hyperfine Interactions in Molecular Iodine," Phys. Rev. A 6 1, pp. 10-20 (1972).

6. D. J. Ruben, et. al. "Laser-Molecular Beam Measurement of Hyperfine Structure in the $\mathrm{I}_{2}$ Spectrum," Chem. Phys. Lett. 22 , pp. 326-330 (1973).

7. H. Ashkenas, and F. S. Sherman, "The Structure and Utilization of Supersonic Free Jets in Low Density Wind Tunnels" in Advances in Applied Mechanics, Supplement 3, Vol. 2, Academic Press, New York, 1966.

8. C. E. Glass, Research scientist, NASA Langley Research Center, Hampton, Virginia. Personal communication.

9. F. Robben and L. Talbot, "Measurement of Shock Wave Thickness by the Electron Beam Fluorescence Method", Phys. Fluids 94, pp. 633-643 (1966). 